\title{
Observing exoplanets with the JWST NIRCam grisms
}

\section{Thomas Greene, Charles Beichman, Daniel Eisenstein, Scott Horner, Douglas Kelly, et al.}

Thomas Greene, Charles Beichman, Daniel Eisenstein, Scott Horner, Douglas Kelly, Yalan Mao, Michael Meyer, Marcia Rieke, Fang Shi, "Observing exoplanets with the JWST NIRCam grisms," Proc. SPIE 6693, Techniques and Instrumentation for Detection of Exoplanets III, 66930G (19 September 2007); doi: 10.1117/12.732506

Event: Optical Engineering + Applications, 2007, San Diego, California, United States 


\title{
Observing Exoplanets with the JWST NIRCam grisms
}

\author{
Thomas Greene $^{a}$, Charles Beichman ${ }^{b}$, Daniel Eisenstein ${ }^{c}$, Scott Horner ${ }^{d}$, Douglas Kelly ${ }^{c}$, \\ Yalan Mao ${ }^{d}$, Michael Meyer ${ }^{c}$, Marcia Rieke ${ }^{c}$, and Fang Shi ${ }^{e}$ \\ ${ }^{a}$ NASA's Ames Research Center, MS 245-6, Moffettt Field, CA 94062; \\ ${ }^{b}$ IPAC / MSC, Caltech 100-22, Pasadena, CA, 91125; \\ ${ }^{c}$ Steward Observatory, University of Arizona, Tucson, AZ 85721; \\ ${ }^{d}$ Lockheed Martin Advanced Technology Ctr., 3251 Hanover St., Palo Alto, CA 94304; \\ ${ }^{e}$ Jet Propulsion Laboratory, 4800 Oak Grove Drive, Pasadena, CA 91109
}

\begin{abstract}
The near-infrared camera (NIRCam) on the James Webb Space Telescope (JWST) will incorporate 2 identical grisms in each of its 2 long wavelength channels. These transmission gratings have been added to assist with the coarse phasing of the JWST telescope, but they will also be used for slitless wide-field scientific observations over selectable regions of the $\lambda=2.4-5.0 \mu \mathrm{m}$ wavelength range at spectroscopic resolution $R \equiv \lambda / \delta \lambda \simeq 2000$. We describe the grism design details and their expected performance in NIRCam. The grisms will provide pointsource continuum sensitivity of approximately $\mathrm{AB}=23 \mathrm{mag}$ in $10,000 \mathrm{~s}$ exposures with $\mathrm{S} / \mathrm{N}=5$ when binned to $R=1000$. This is approximately a factor of 3 worse than expected for the JWST NIRSpec instrument, but the NIRCam grisms provide better spatial resolution, better spectrophotometric precision, and complete field coverage. The grisms will be especially useful for high precision spectrophotometric observations of transiting exoplanets. We expect that $R=500$ spectra of the primary transits and secondary eclipses of Jupiter-sized exoplanets can be acquired at moderate or high signal-to-noise for stars as faint as $\mathrm{M}=10-12 \mathrm{mag}$ in $1000 \mathrm{~s}$ of integration time, and even bright stars $(\mathrm{V}=5 \mathrm{mag})$ should be observable without saturation. We also discuss briefly how these observations will open up new areas of exoplanet science and suggest other unique scientific applications of the grisms.
\end{abstract}

Keywords: NIRCam, grisms, JWST, near-infrared, exoplanets, spectroscopy

\section{INTRODUCTION}

The James Webb Space Telescope (JWST) is being designed and developed to be a large observatory (6.5-m diameter primary mirror) that will be operated at cold temperatures $(\mathrm{T}<40 \mathrm{~K})$ with exquisite sensitivity over near- and mid-infrared (IR) wavelengths. The primary mirror will consist of 18 hexagonal segments that will be deployed from stowed positions after launch and will be co-phased so that the telescope provides diffractionlimited performance at near-IR and longer wavelengths. The primary coarse segment phasing approach is to insert arrays of dispersed Hartmann sensor (DHS) elements in the NIRCam ${ }^{1}$ instrument's pupil wheel while pointed at a bright star, analyze the resultant data on the ground, command movements of the segments, and repeat the process until the segments are within the range of the fine phasing process ${ }^{2}$ (200 nm phase step errors).

The JWST project reviewed this coarse phasing approach and decided to augment it with a secondary approach that would provide a larger capture range, allowing the segments to have larger initial piston errors. This Dispersed Fringe Sensing (DFS) technique involves using a monolithic transmission grating (or grism) with a clear aperture large enough to accommodate the entire telescope pupil (the image of all primary segments), whereas each DHS element spans only the edges of adjacent segments. Two identical grisms are used in 2 pupil wheel positions, with the grisms rotated 90 degrees apart so that either of 2 perpendicular dispersion directions can be selected. The DFS and DHS approaches use the same fringe analysis algorithm to sense the wavefront piston, and the DFS approach can also directly measure segment piston without global reconstruction; it is

Further author information: (Send correspondence to T.G.)

T.G.: E-mail: thomas.p.greene@nasa.gov, Telephone: 16506045520

Techniques and Instrumentation for Detection of Exoplanets III, edited by Daniel R. Coulter

Proc. of SPIE Vol. 6693, 66930G, (2007) · 0277-786X/07/\$18 · doi: 10.1117/12.732506

Proc. of SPIE Vol. 6693 66930G-1 
robust against severe segment displacement errors. The DFS technique has been modeled and demonstrated to have perform well for JWST. ${ }^{3}$

The DFS grisms can also be used for scientific observations, providing near-IR slitless spectra when used in NIRCam (similar to the grisms in the NICMOS Hubble Space Telescope instrument; see http://www.stsci.edu/hst /nicmos/performance/spectroscopy). The NIRSpec JWST instrument is a capable multi-object near-IR spectrograph, but slitless grism observations with NIRCam provide advantages in several applications. First, even the NIRCam longwave channel has a finer pixel scale than NIRSpec (0.065 vs. 0.100 arcseconds per pixel), so NIRCam would be capable of making higher spatial resolution spectroscopic observations. The NIRCam longwave channel critically samples the JWST diffraction-limited PSF at $\lambda=4.0 \mu \mathrm{m}$. Second, slitless spectra would not suffer from slit losses, so high spectrophotometric precision observations can be made with NIRCam even in the presence of pointing drifts or jitter. Third, the ability to dither the slit-less spectra across the NIRCam detector should allow for considerably better flat-fielding than staring with NIRSPEC. Fourth, NIRCam slitless spectra completely sample the entire NIRCam field $\left(2.2^{\prime} \times 4.4^{\prime}\right.$ for both modules), whereas the NIRSpec multi-object spectrograph operates through a microshutter array ${ }^{4}$ that obscures a fraction of the NIRSpec field (9.7 square arc-minutes total field). Slitless NIRCam spectra also have two primary disadvantages compared to NIRSpec. First, NIRSpec is more sensitive because its multishutter array blocks most of the astronomical background. Second, Slitless NIRCam spectra may overlap and become confused in crowded regions where many sources are present, whereas this can be prevented with NIRSpec's microshutter array.

\section{GRISM DESIGN}

The grism design is driven by the wavefront sensing requirements of achieving maximum segment piston capture range and good resolution of segment piston positions (small minimum detectable piston). Large capture range drives the design to producing spectra at the longest possible wavelengths, and a small minimum detectable piston drives the design toward low spectroscopic resolution. These effects are coupled such that low spectral resolutions provide small minimum detectable pistons, but their relatively small short wavelength cut-ons reduce the maximum piston capture range.

These factors drove the long wavelength grism cutoff to be equal to NIRCam's long wavelength cutoff, $\lambda=$ $5.0 \mu \mathrm{m}$. The adopted design also specifies that the spectroscopic resolution be $R \equiv \lambda / \delta \lambda \simeq 2000$, producing a central undeviated wavelength $\lambda=4.0 \mu \mathrm{m}$ and a short wavelength cutoff $\lambda=3.0 \mu \mathrm{m}$ on NIRCam's $2048 \times$ 2048 pixel longwave detector array (with 2 pixels per resolution element). The large operating wavelength range dictates that the grisms operate in first order, $\mathrm{m}=1$.

These nominal wavelength cutoffs and undeviated wavelength are realized only when a source is located at the center of the field of view. Displacing a source toward the long-wavelength dispersion direction causes wavelengths shorter than $3.0 \mu \mathrm{m}$ to strike the short wavelength end of the detector and wavelengths shorter than $5.0 \mu \mathrm{m}$ to strike the long wavelength end of the detector. Likewise, displacing a source toward the shortwavelength dispersion direction causes wavelengths longer than $3.0 \mu \mathrm{m}$ to strike the short wavelength end of the detector and wavelengths longer than $5.0 \mu \mathrm{m}$ to strike the long wavelength end of the detector. However, the detector is not sensitive to wavelengths longer than about $5.0 \mu \mathrm{m}$.

The grisms will be installed in the pupil wheels of only the NIRCam longwave channels, which operate over $\lambda=2.4-5 \mu \mathrm{m}$ ( 2 identical grisms in each of the 2 identical longwave channels). The NIRCam pupil wheels (located at the actual system pupil) are in series with filter wheels, so the grisms must be used in series with a filter (also needed to reject higher spectral orders). Table 1 gives the wavelength cutoffs and spectral lengths for continuum sources located within the field such that both the short $(\lambda 1)$ and long $(\lambda 2)$ wavelength limits fall on the detector for several filters. ${ }^{5}$ See also http://ircamera.as.arizona.edu/nircam/ for a complete filter list and other NIRCam information.

The adopted grism design provides minimum and maximum detectable wavefront error of approximately 0.12 $\mu \mathrm{m}$ and $291 \mu \mathrm{m}$ in piston between 2 segments, somewhat dependent on which filters are used. Errors larger than this maximum piston can be detected directly from the telescope point spread function.

Several practical issues also drove the grism design. First, the NIRCam pupil and filter wheels are spaced closely, separated by only about $10 \mathrm{~mm}$. The elements in these wheels are also large, with $48 \mathrm{~mm}$ diameters and 
Table 1. Ranges of grism spectra.

\begin{tabular}{|l|r|r|r|r|}
\hline Filter & $\lambda 1$ & $\lambda 2$ & \# pixels & \# pixels/2048 \\
\hline F277W & 2.42 & 3.12 & 696 & 0.34 \\
\hline F322W2 & 2.42 & 4.03 & 1600 & 0.78 \\
\hline F356W & 3.12 & 4.01 & 885 & 0.43 \\
\hline F410M & 3.90 & 4.31 & 408 & 0.20 \\
\hline F444W & 3.89 & 5.00 & 1104 & 0.54 \\
\hline
\end{tabular}

Table 2. Basic grism specifications

\begin{tabular}{|l|l|}
\hline Material & Optical grade monocrystalline Si \\
\hline Operating temperature & $\sim 35 \mathrm{~K}$ \\
\hline groove frequency & $65 \mathrm{~mm}^{-1}$ \\
\hline prism angle & $6.16^{\circ}$ \\
\hline blaze angle & $5.75^{\circ}$ \\
\hline maximum thickness & $10.0 \mathrm{~mm}$ \\
\hline Diameter & $48.0 \mathrm{~mm}$, circular \\
\hline Clear Aperture & $42.0 \mathrm{~mm}$, circular \\
\hline AR coating & $2.4-5 \mu \mathrm{m}$, both sides \\
\hline
\end{tabular}

$42 \mathrm{~mm}$ clear apertures. This requires the grism to have a small prism angle, less than about 10 degrees. Given this small angle, the relatively high resolution $(R \simeq 2000)$ dictates a relatively large refractive index, $n \gtrsim 2.5$, as derived from standard first-order grism design equations. ${ }^{6}$ The grism material must also maintain high and uniform optical transmission after exposure to a significant dose of cosmic radiation over the lifetime of JWST. These constraints drove us to select monocrystalline $\mathrm{Si}$ as the grism material.

The blaze angle was chosen to maximize efficiency near $\lambda=3.7 \mu \mathrm{m}$, the average of all wavelengths to be observed with the grisms for sources located anywhere in the field. This is slightly different from the undeviated wavelength $(\lambda=4.0 \mu \mathrm{m})$, so the blaze angle is offset slightly from the prism angle. The grisms are now being procured, and their basic manufacturing specifications are given in Table 2.

\section{ESTIMATED PERFORMANCE}

\subsection{Optical Performance}

Several aspects of the grism performance have been modeled or estimated. The grism blaze efficiency was calculated with PCGRATE (http://www.pcgrate.com/). The peak first order blaze efficiency was found to be 0.87 assuming zero reflective losses (perfect AR coatings on both the flat entrance face and grooves), with the blaze peak located very close to the $\lambda=3.7 \mu \mathrm{m}$ specification used for the first order design. The efficiency is greater than or equal to 0.83 times the peak for $3.1-5.0 \mu \mathrm{m}$ wavelengths (the primary grism range) and falls to 0.4 times the peak at the $\lambda=2.4 \mu \mathrm{m}$, the short wavelength cutoff of the NIRCam longwave channels. These efficiencies assume perfect sawtooth grating profiles, and the actual absolute efficiency is likely to be somewhat lower because of imperfect groove profiles and AR coatings.

The imaging performance of the grisms was modeled by adding surfaces representing the grism to the Code $\mathrm{V}$ optical model of NIRCam. The layout of this configuration is shown in Figure 1. Code V was then used to verify the wavelength coverage and measure the quality of NIRCam images with the grism in place. Figure 2 shows that the wavelength locations and dispersion are very close to the predictions of the first order design; the wavelengths $3.0-5.0 \mu \mathrm{m}$ are imaged onto the detector, and the central undeviated wavelength is $\lambda=4.0 \mu \mathrm{m}$. 

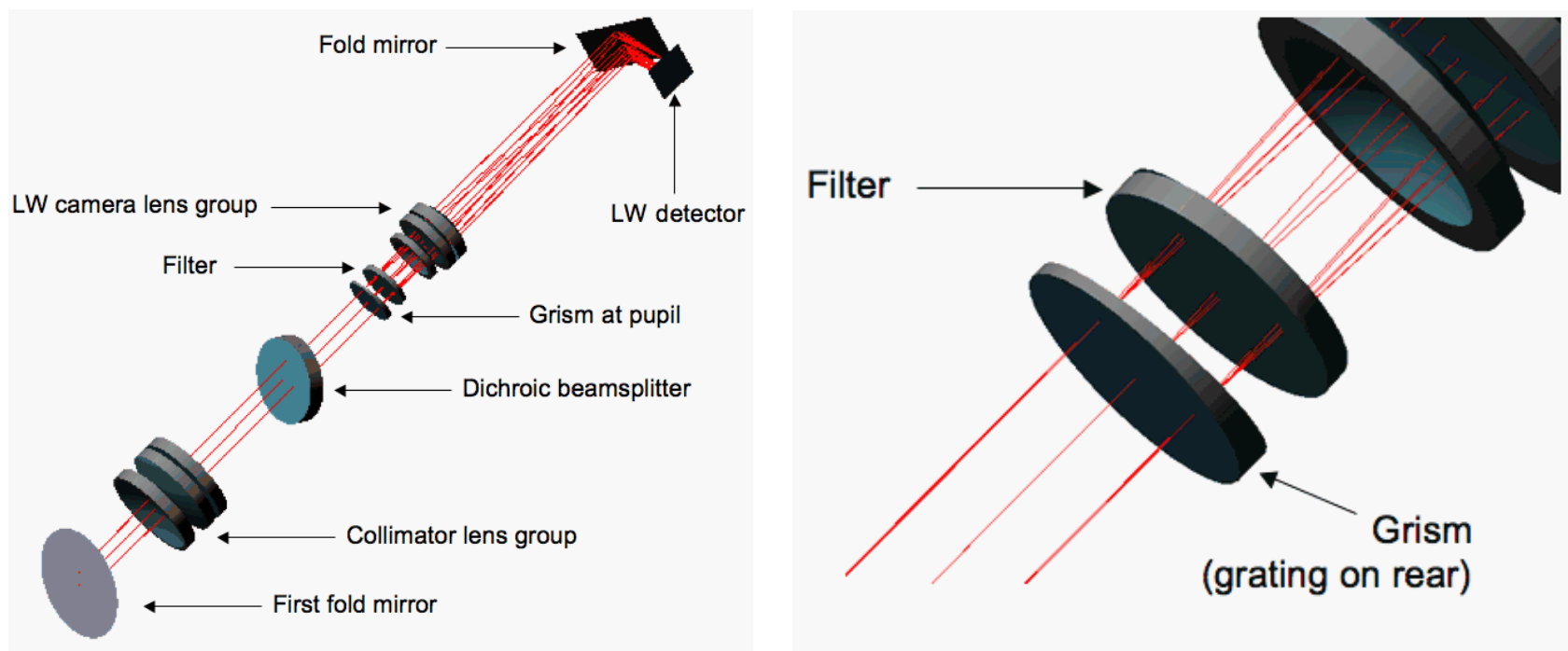

Figure 1. Optical layout of a NIRCam longwave (LW) channel with a grism. Left panel: Optical components are indicated, and light enters NIRCam from the first fold mirror at the bottom left. Right panel: Detail of the grism and filter region. Incoming light strikes the flat entrance face of the grism; its grating surface is on the rear side.
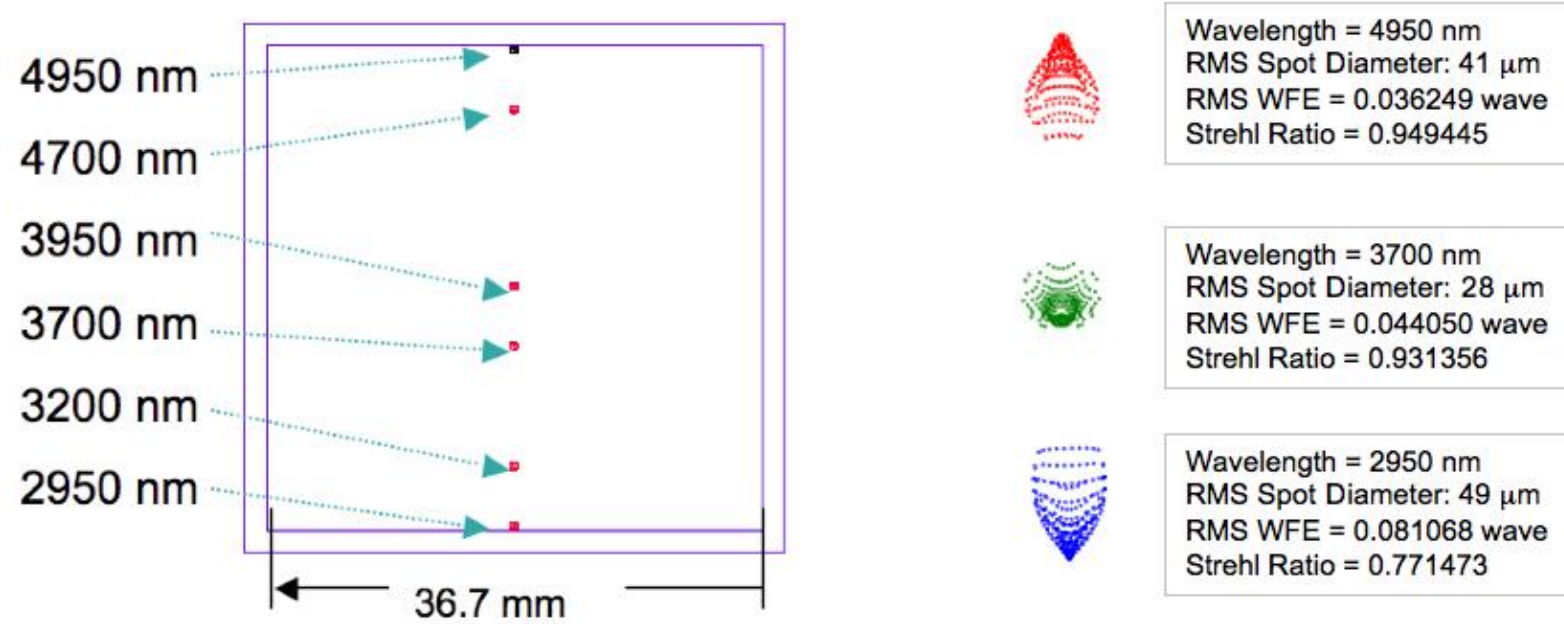

Wavelength $=3700 \mathrm{~nm}$

RMS Spot Diameter: $28 \mu \mathrm{m}$

RMS WFE $=0.044050$ wave

Strehl Ratio $=0.931356$

Wavelength $=2950 \mathrm{~nm}$

RMS Spot Diameter: $49 \mu \mathrm{m}$

RMS WFE $=0.081068$ wave

Strehl Ratio $=0.771473$

Figure 2. Optical performance of a NIRCam longwave (LW) channel with a grism for a point source in the center of its field. The left side shows that the wavelengths $3.0-5.0 \mu \mathrm{m}$ are imaged onto the detector with a central undeviated wavelength $\lambda=4.0 \mu \mathrm{m}$ as prescribed in the first order design. The right side shows the optical spot pattern and image quality measures at 3 wavelengths at the edges and center of the detector. The spot sizes compare favorably to the width of 2 detector pixels $(36 \mu \mathrm{m})$.

This figure also shows that NIRCam has good image quality with the grism for a source located in the center of the field of view. RMS spot sizes are approximately equal to the width of 2 pixels (where each pixel is 18 $\mu \mathrm{m}$ in size), indicating that the grisms will achieve close to their predicted spectroscopic $(R \simeq 2000)$ and spatial resolutions. We have also determined that good image quality ( $\sim 2$ pixel spots) is maintained over much of the entire field for wavelengths $\lambda \gtrsim 3 \mu \mathrm{m}$. However, image quality degrades somewhat at shorter wavelengths, with RMS spot sizes reaching approximately 4 pixels at the $\lambda=2.4 \mu \mathrm{m}$ cutoff. Only objects near 1 edge of the field will have spectra at such short wavelengths, and their resolutions will be degraded as indicated by the spot sizes. 
Table 3. Sensitivities of NIRCam grism observations for $\mathrm{t}=10,000 \mathrm{~s}$ total real time observations for $\mathrm{S} / \mathrm{N}=5$ and binned to $R=1000$. See text for further explanation.

\begin{tabular}{|l|r|r|r|r|r|}
\hline Filter & F277W & F322W & F356W & F410M & F444W \\
\hline$\lambda_{\text {ctr }}(\mu \mathrm{m})$ & 2.77 & 3.22 & 3.56 & 4.10 & 4.44 \\
\hline$F_{\text {cont }}(\mu \mathrm{Jy})$ & 2.0 & 2.5 & 1.8 & 1.7 & 3.3 \\
\hline AB mag & 23.2 & 22.9 & 23.3 & 23.4 & 22.6 \\
\hline$F_{\text {line }}\left(10^{-15} \mathrm{~W} \mathrm{~m}^{-2}\right)$ & 4.4 & 4.7 & 3.1 & 2.5 & 4.5 \\
\hline$F_{\text {line }}\left(10^{-18} \mathrm{erg} \mathrm{s}^{-1} \mathrm{~cm}^{-2}\right)$ & 4.4 & 4.7 & 3.1 & 2.5 & 4.5 \\
\hline
\end{tabular}

\subsection{Sensitivity Estimates}

Point-source sensitivity estimates for the NIRCam grisms were derived from the NIRCam imaging sensitivity model spreadsheet obtained from M. Rieke, the NIRCam PI. The background, throughput, and detector performance information contained in this spreadsheet are given in the document JWST-CALC-003894, JWST NIRCam Sensitivity Calculations, available at http://www.stsci.edu/jwst/externaldocs/technicalreports/JWSTSTScI-001007.pdf. The NIRCam point source imaging sensitivity values were scaled from the spreadsheet calculations after adopting a conservative peak transmission efficiency factor of 0.60 for the grism and applying an approximate blaze function shape matching the PCGRATE results. Sensitivities were calculated assuming that background photons over the entire bandpass of the selected filter are collected in each detector pixel, but the radiation from a point source has a bandwidth of only $\lambda / 4000$ per pixel. No penalty for source confusion was applied. The NIRCam photometric sensitivities in the spreadsheet model were calculated for circular apertures 5 pixels $\left(0.33^{\prime \prime}\right)$ in diameter, considerably larger than the diffraction-limited resolution size at most wavelengths. Therefore an effective resolution size of 4 spectral pixels $(R=1000)$ and 5 spatial pixels was chosen for deriving the sensitivities shown in Table 3.

The value $F_{\text {cont }}$ in Table 3 is the point source continuum sensitivity in $\mu \mathrm{Jy}$ for a $5 \sigma$ detection in $10,000 \mathrm{~s}$ total real time $(\sim 9500 \mathrm{~s}$ total integration time). This continuum point source sensitivity is also given in $\mathrm{AB}$ magnitudes. The two $F_{\text {line }}$ values are sensitivities to unresolved emission lines integrated over a $4 \times 5$ pixel, $\mathrm{R}=1000$ resolution element.

JWST NIRSPEC is predicted to have a continuum sensitivity of $630 \mathrm{nJy}$ for multi-object spectroscopy at $R=1000$ for a $10,000 \mathrm{~s}$ total exposure with $\mathrm{S} / \mathrm{N}=5$ in the $\lambda=4.0 \mu \mathrm{m}$ region. This is about $3-4$ times better than using the NIRCam grisms, so equivalent NIRCam observations would take 10 times longer or more. Sensitivity to unresolved line flux also scales in similar fashion. However, the NIRCam grisms are more competitive with NIRSpec at shorter integration times because NIRSpec is always detector noise limited at $R=1000$ and NIRCam is detector noise limited only for short exposures, $t \lesssim 100 \mathrm{~s}$. The continuum sensitivities of both NIRCam and NIRSpec should both be about $9 \mu \mathrm{Jy}$ for $\mathrm{S} / \mathrm{N}=5$ with a total of $500 \mathrm{~s}$ integration time consisting of 5 separate $100 \mathrm{~s}$ exposures. NIRCam does not achieve greater advantage for shorter exposure times $t<100 \mathrm{~s}$ because then NIRCam also becomes detector-noise limited (except in the very wide F322W2 filter which does not become detector-noise limited until $t \sim 50 \mathrm{~s}$ ).

\subsection{Saturation Limits}

The high dispersion of the grisms allow observations of very bright objects despite NIRCam's relatively long $10.6 \mathrm{~s}$ full frame integration time and modest detector full well requirement of $7.2 \times 10^{4}$ electrons. The NIRCam point source imaging saturation limits were scaled by the transmission efficiency and dispersion of the grisms, and this resulted in saturation limits of $500 \mathrm{mJy}-1 \mathrm{Jy}$ in the 5 medium and wide band filters that are listed in Table 3. This allows observation of solar-type stars as bright as $\mathrm{K}=6-7.4 \mathrm{mag}(9.0-9.7 \mathrm{AB} \mathrm{mag})$ to be observed without saturation. Solar-type stars as bright as $\mathrm{K}=3.6 \mathrm{mag}(\mathrm{V}=5.0 \mathrm{mag})$ can be observed with any grism / filter combination without saturation when using a standard $1024 \times 16$ pixel subarray detector readout at its corresponding minimum integration time of 0.32 seconds. 


\section{TRANSITING EXOPLANET OBSERVATIONS WITH THE NIRCAM GRISMS}

The NIRCam grisms are particularly well suited for observing transiting extrasolar planets. Transiting exoplanets provide the opportunity to separate the flux from an exoplanet and its parent star by making sensitive differential measurements at appropriate times (or phases) of an exoplanet's orbit. ${ }^{7}$ Much is being learned about extrasolar planets from precision photometric and spectroscopic observations as they transit and are eclipsed by their host stars. This is especially powerful for transiting exoplanets with known radial velocities (RVs), as the combination of transit and RV data yields planetary masses, radii, densities, orbital distances (and periods), and potentially compositions. NIRCam transiting exoplanet targets will come from radial velocity and ground-based transit surveys, from space-based transit missions such as Kepler and CoRoT, and from surveys by JWST itself of small regions of special interest such as globular clusters. Potential follow-up opportunities with JWST/NIRCam will include precision photometry to search for rings and moons, transit timing to look for orbital perturbations by other massive bodies in the planetary system, and determination of light curves of non-transiting RV systems. In addition, the NIRCam grisms offer the exciting prospect of making these observations at spectral resolutions $R=$ 500 - 2000 with signal-to-noise high enough to measure numerous important spectral features and atmospheric constituents over the $\lambda=2.4-5 \mu \mathrm{m}$ region. This section summarizes some of the opportunities and potential problems with NIRCam grism observations of transits and light curves.

\subsection{Host Stars and Observational Parameters}

The magnitudes of potential target stars (Table 4) range from the very bright for planets found in RV studies or shallow, wide-angle transit surveys to the very faint for narrow angle transit surveys such as OGLE or with JWST itself. Potential spectral types of host stars range from $\mathrm{G}$ stars to $\mathrm{M}$ dwarf stars with the latter being particularly interesting because the small size of the star makes possible the detection of very small planets, possibly even as small as the Earth! Table 4 lists Johnson magnitudes from $\mathrm{V}=5-20$ mag and for calculations with the NIRCam grism, from $\mathrm{M}=3-18$ mag. In what follows we use M band (F440W filter wavelength) magnitudes of 5, 10 and 15 mag to probe the capabilities of grism observations.

Table 4. Potential exoplanet host star targets

\begin{tabular}{|l|l|l|}
\hline Technique & $\begin{array}{l}\text { GV Star Brightness } \\
\text { (Johnson mag) }\end{array}$ & $\begin{array}{l}\text { M5V Star Brightness } \\
\text { (Johnson mag) }\end{array}$ \\
\hline Radial Velocity & $\mathrm{V}=5-10$ & $\mathrm{~V}=8-10$ \\
& $\mathrm{M}=3.5-8.5$ & $\mathrm{M}=3-5$ \\
\hline Ground transit & $\mathrm{V}=5-19$ & $\mathrm{~V}=5-19$ \\
& $\mathrm{M}=3.5-17.5$ & $\mathrm{M}=3-14$ \\
\hline $\begin{array}{l}\text { Space Transit } \\
\text { (Kepler/CoRoT) }\end{array}$ & $\mathrm{V}=9-15$ & $\mathrm{~V}=9-15$ \\
\hline JWST Transits & $\mathrm{M}=7.5-13.5$ & $\mathrm{M}=4-10$ \\
(e.g. 47 Tuc) & $\mathrm{V}=15-20$ & $\mathrm{~V}=20$ \\
\hline
\end{tabular}

To proceed further we estimate the flux contrast ratios for various star/planet combinations for both primary and secondary transits. In Table 5 the depth of the primary transit is the ratio of the appropriate planet $(1$ $\mathrm{R}_{\mathrm{Jup}}$ and $\left.1 \mathrm{R}_{\oplus}\right)$ to stellar $\left(1 \mathrm{R}_{\odot}\right.$ and $\left.0.5 \mathrm{R}_{\odot}\right)$ areas. The estimate for the secondary eclipse uses the same areal ratios times the ratio of blackbody emission from the star and planet where we have assumed planet emission of a $1500 \mathrm{~K}$ blackbody and stellar temperatures of $5500 \mathrm{~K}$ and $3000 \mathrm{~K}$. As Figure 3 suggests, a blackbody is an overly simple characterization of a planet's spectrum, but we use it here simply for convenience.

The signal-to-noise ratio (SNR) estimates are based on number of detected photo-electrons from the starplanet system assuming an overall throughput of 0.20 , an integration time $t$, and a resolving power $R=500$ (obtained by binning over $\sim 8$ spectral pixels) which will be appropriate for all but the brightest systems. Lower resolutions may be needed for fainter stars and the SNR can be estimated by scaling by $\sqrt{500 / R}$. The relevant 
Table 5. Transit flux ratios at $\lambda=4.4 \mu \mathrm{m}$

\begin{tabular}{|l|r|r|r|r|}
\hline & \multicolumn{2}{|c|}{ Primary Transit* } & \multicolumn{2}{c|}{ Secondary Eclipse** } \\
\hline & G2 V Star & \multicolumn{1}{c|}{ M5 V Star } & G2 V Star & \multicolumn{1}{c|}{ M5 V Star } \\
\hline Jupiter & 0.010 & 0.160 & 0.0010 & 0.040 \\
\hline Earth & $8.4 \times 10^{-5}$ & 0.00134 & $8.7 \times 10^{-6}$ & 0.00034 \\
\hline & $\begin{array}{l}\text { *Ratio of areas of planets to } \\
1.0 \text { and } 0.5 \mathrm{R}_{\text {Sun }} \text { stars }\end{array}$ & $\begin{array}{l}\text { **Ratio of areas times ratio } \\
\text { of 1500K blackbody planet to } \\
5500 \mathrm{~K} \text { or 3000 K star. }\end{array}$ \\
\hline
\end{tabular}

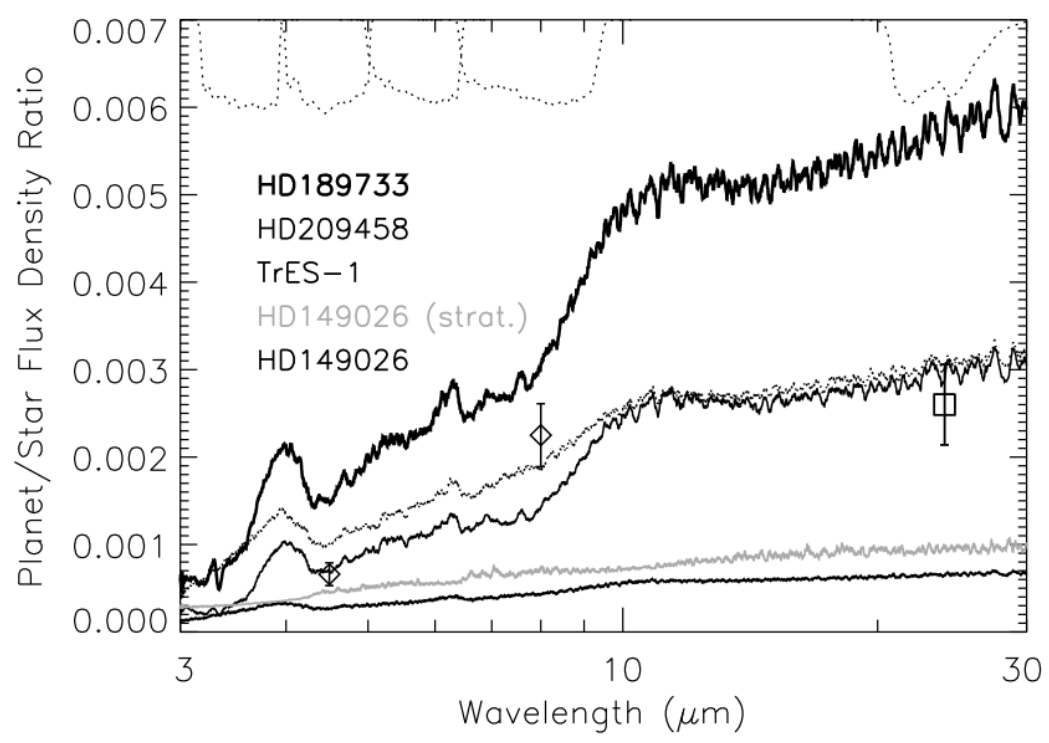

Figure 3. Spitzer photometry of a number of transiting systems (diamonds, TrES-1; square, HD 209458b) compared with theoretical models. ${ }^{8}$

measurable quantity is $\phi t($ Planet $)= \pm[\phi t($ Star \pm Planet $)-\phi t($ Star $)]$ where the signal, $\phi$, is measured in photo-electrons and the sign depends whether the event is a secondary eclipse or primary transit. The quantity $\phi t$ (Planet) is obtained by subtracting two grism datasets composed of averages of spectra measured in and out of eclipse. The noise in the measurement can be estimated from the root-sum-square (RSS) combination of the two main noise sources in each image: photon noise from $\sqrt{2[\phi t(\text { Star })+\phi t(\text { Background })]}$ and the "flat-field" noise $\beta[\phi t($ Star $)+\phi t($ Background $)$ where $\beta$ is a parameter which quantifies how well one can remove drifts, common-mode noise sources, pixel-to-pixel variations, etc. The factor of two comes from the fact that we must difference two spectra to get the signal of the exoplanet.

\subsection{Data Quality Expectations}

Table 6 provides SNRs in integration times of $t=1000 \mathrm{sec}$ for three limiting cases: 1$)$ bright stars $(\mathrm{M}=5$ mag; $\phi=8 \times 10^{4} \mathrm{elec} \mathrm{sec}^{-1}$ at full $R=2000$ resolution) where flat field performance is critical compared with shot noise; 2) moderately bright stars $\left(\mathrm{M}=10 \mathrm{mag} ; \phi=800 \mathrm{elec} \mathrm{sec}^{-1}\right)$ where both flat field and photon noise are important; and 3) faint stars $\left(\mathrm{M}=15 \mathrm{mag} ; \phi=8 \mathrm{elec} \mathrm{sec}^{-1}\right)$ which are dominated by photon noise. It is important to note that in all these cases the dominant photon noise comes the star since the observatory background of under $2 \mathrm{elec} \mathrm{sec}^{-1}$ in the F440W filter is small for even the faintest stars. Read noise is negligible. Saturation effects will have to be considered for bright sources, but integration times of $1-5$ sec should be adequate for $\mathrm{M}=5 \mathrm{mag}$ sources once the spreading of light over a number of spatial and spectral pixels is taken into account. These and brighter stars can be observed easily in sub-array mode which allows very short integration times for subregions of the detector. 
Table 6. SNRs for transits and eclipses of G2V and M5V stars $\left(\mathrm{T}_{\text {planet }} \simeq 1500 \mathrm{~K}\right)$.

\begin{tabular}{|c|c|c|c|c|c|c|c|}
\hline \multicolumn{8}{|c|}{ SNR for Primary Transit of G2V Star. Resolution $=500^{*} ; \tau=1,000$ sec } \\
\hline Jupiter & & $M(m a g)^{* *}$ & & Earth & & $M(\mathrm{mag})$ & \\
\hline Flat/mag & 5 & 10 & 15 & Flat/mag & 5 & 10 & 15 \\
\hline 1.0E-06 & 88.32 & 8.83 & 0.82 & 1.0E-06 & 0.74 & 0.07 & 0.01 \\
\hline 1.0E-05 & 87.65 & 8.82 & 0.82 & 1.0E-05 & 0.73 & 0.07 & 0.01 \\
\hline 1.0E-04 & 55.37 & 8.76 & 0.82 & 1.0E-04 & 0.46 & 0.07 & 0.01 \\
\hline $1.0 \mathrm{E}-03$ & 7.08 & 5.54 & 0.81 & $1.0 \mathrm{E}-03$ & 0.06 & 0.05 & 0.01 \\
\hline \multicolumn{8}{|c|}{ SNR for Secondary Eclipse of G2V Star. Resolution= $500 ; \tau=1,000 \mathrm{sec}$} \\
\hline Jupiter & & $M($ mag $)$ & & Earth & & $M($ mag) & \\
\hline Flat/mag & 5 & 10 & 15 & Flat/mag & 5 & 10 & 15 \\
\hline 1.0E-06 & 9.12 & 0.91 & 0.09 & $1.0 \mathrm{E}-06$ & 0.08 & 0.01 & 0.00 \\
\hline 1.0E-05 & 9.05 & 0.91 & 0.09 & 1.0E-05 & 0.08 & 0.01 & 0.00 \\
\hline 1.0E-04 & 5.71 & 0.90 & 0.09 & 1.0E-04 & 0.05 & 0.01 & 0.00 \\
\hline $1.0 \mathrm{E}-03$ & 0.73 & 0.57 & 0.09 & 1.0E-03 & 0.01 & 0.00 & 0.00 \\
\hline
\end{tabular}

\begin{tabular}{|c|c|c|c|c|c|c|c|}
\hline \multicolumn{8}{|c|}{ SNR for Primary Transit of M5V Star. Resolution= $500 ; \tau=1,000 \mathrm{sec}$} \\
\hline Jupiter & & $M($ mag) & & Earth & & $M($ mag) & \\
\hline Flat/mag & 5 & 10 & 15 & Flat/mag & 5 & 10 & 15 \\
\hline 1.0E-06 & 355.97 & 35.60 & 3.56 & 1.0E-06 & 2.96 & 0.30 & 0.03 \\
\hline 1.0E-05 & 353.32 & 35.60 & 3.56 & 1.0E-05 & 2.94 & 0.30 & 0.03 \\
\hline $1.0 \mathrm{E}-04$ & 224.16 & 35.33 & 3.56 & $1.0 \mathrm{E}-04$ & 1.85 & 0.29 & 0.03 \\
\hline $1.0 \mathrm{E}-03$ & 28.76 & 22.42 & 3.53 & 1.0E-03 & 0.24 & 0.19 & 0.03 \\
\hline \multicolumn{8}{|c|}{ SNR for Secondary Eclipse of M5V Star. Resolution= $500 ; \tau=1,000 \mathrm{sec}$} \\
\hline Jupiter & & $\bar{M}$ (mag) & & Earth & & $M($ mag) & \\
\hline Flat/mag & 5 & 10 & 15 & Flat/mag & 5 & 10 & 15 \\
\hline 1.0E-06 & 88.45 & 8.85 & 0.88 & 1.0E-06 & 0.75 & 0.07 & 0.01 \\
\hline 1.0E-05 & 87.77 & 8.84 & 0.88 & 1.0E-05 & 0.74 & 0.07 & 0.01 \\
\hline 1.0E-04 & 55.28 & 8.78 & 0.88 & $1.0 \mathrm{E}-04$ & 0.47 & 0.07 & 0.01 \\
\hline 1.0E-03 & 7.06 & 5.53 & 0.88 & 1.0E-03 & 0.06 & 0.05 & 0.01 \\
\hline
\end{tabular}

Examination of Table 6 shows that in the photon noise limit (flat field noise coefficient $\beta \leq 10^{-5}$ ), it is possible to achieve very high SNR on Jupiter transits, SNR $=50-100$ for GV stars and $100-300$ for MV stars, in each spectral resolution element for transits seen against $\mathrm{M}=5 \mathrm{mag}$ bright stars. It would even possible to detect the spectrum of an Earth-sized planet, albeit one that could hardly be called habitable at a temperature of $1500 \mathrm{~K}$. Overall, this spectroscopic performance will enable high quality observations of the wide variety of transiting planets that will be known by the time JWST flies. These data will place fundamental constraints on contemporary theories at much more stringent levels compared to present day results from Spitzer (Figure 3).

However, for $\beta \geq 10^{-5}$, the effects of various non-photon noise sources become critical, and $\beta \sim 10^{-3}$ can render the transit undetectable. NIRCam's actual performance will depend on the repeatability and stability of the NIRCam detector arrays, detector electronics, optical artifacts (including any fringing produced by the grisms), as well as the calibration scheme used for the observations. Spitzer results show that $\beta=10^{-4}$ is possible for IRAC and MIPS photometry, but ongoing efforts to push below $\beta=10^{-3}$ for IRS spectra have proven difficult. ${ }^{9}$ Dithering slitless NIRCam grism observations may be able to reduce flat-field noise to below that of the Spitzer IRS. We will need to monitor and remove gain fluctuations of the form $G(\lambda$, time, pixel $)$ to reach the best performance listed in the table. Calibration steps may include: 1) observations of stars of similar spectral type observed as close in time as possible to the transit; 2) spatial dithering to help average over pixel-to-pixel variations; and 3) extracting spectra for other sources in the NIRCam frame to monitor commonmode gain drifts. Success in pushing to low values of $\beta$ will depend on the extent that the gain fluctuations are separable into $G(\lambda$, time, pixel $)=G_{1}(\lambda$, pixel $) G_{2}($ time, pixel $)$ functions that can be determined from the data. In this regard, the intrinsic multi-object nature of the NIRCam grism will offer many advantages over existing 
Spitzer datasets. The NIRCam test program should include procedures to help us to assess the stability of the grism mode.

\section{NEW SCIENCE FRONTIERS}

We have described how NIRCam grism observations will record high quality near-IR spectra of transiting exoplanets in exposures of under $1000 \mathrm{~s}$. This great leap in observational capability is likely to drive a similarly large leap in exoplanet science. Photometric variations in Spitzer observations have recently been used to derive a surface temperature map for an exoplanet, the hot Jupiter HD 189733b. ${ }^{10}$ High SNR NIRCam grisms observations of this and other similar exoplanets should reveal how the spectra change between the day and night sides of these externally heated planets. It is predicted that the night sides of these planets should show strong $\mathrm{CH}_{4}$ absorptions, while the externally heated day sides should exhibit weak $\mathrm{CH}_{4}$ and strong $\mathrm{CO} .{ }^{11}$ Measurements of these and other features will reveal much about the atmospheric composition and structure of exoplanets as well as provide more insight into how they are affected by their nearby stars.

Time resolved spectra of exoplanets can also be used to make spatial maps of their surfaces and distinguish between their atmospheric models. Depending on orbital parameters, transiting exoplanets can take on the order of $2000 \mathrm{~s}$ to fully disappear behind their parent stars in a secondary eclipse. This would allow on the order of 4 high quality spectroscopic observations, each sampling different regions of the exoplanet's surface or atmosphere. Exoplanet models predict different amounts of limb darkening, and JWST time resolved observations over secondary eclipse should be adequate for measuring this darkening well enough to discriminate between different planetary atmosphere models. ${ }^{12}$ NIRCam grism observations are ideal for these observations because they are free of spectrophotometric errors induced by fixed slits (when any pointing jitter or drifts are present).

The NIRCam grisms are also very well suited for observing resolved planetary mass companions to nearby stars. Ground-based adaptive optics surveys are starting to find spatially resolved planetary mass companions to nearby brown dwarfs. ${ }^{13}$ Numerous faint and presumably very low mass objects have also been found near pre-main-sequence stars in nearby star forming regions. More of both kinds of these objects are likely to be found by the time JWST is launched, and the high sensitivity and spatial resolution of the NIRCam grisms are ideal for obtaining their spectra. Slitless Hubble Space Telescope STIS observations have studied emission line jets from young T Tauri stars, ${ }^{14}$ and NIRCam grism observations of such objects would reveal the sizes, excitations, and column densities of such jets within $\sim 10 \mathrm{AU}$ of nearby $\mathrm{T}$ Tauri stars, probing the radiation environments and gas densities of their planet-forming regions.

Finally, NIRCam grism observations are also idea for wide-field imaging surveys of emission line galaxies. This would allow galaxies to be imaged in high equivalent width (relatively bright) lines, effectively operating as a multi-object integral field unit (IFU). this would be a unique capability for JWST. H $\alpha$, Pa $\alpha$, and other lines are likely to be detectable in many objects, providing redshifts in addition to emission line images. However, we do not discuss any of these applications further since this document is focused on exoplanets.

\section{ACKNOWLEDGMENTS}

We thank K.-W. Hodapp, S. Somerstein, J. Stansberry, and other members of the greater JWST / NIRCam team who have contributed to the NIRCam grism effort in ways outside of the scope of this document.

\section{REFERENCES}

1. M. J. Rieke, D. Kelly, and S. Horner, "Overview of James Webb Space Telescope and NIRCam's Role," Cryogenic Optical Systems and Instruments XI 5904(1), p. 590401, SPIE, 2005.

2. A. Sivaramakrishnan, E. C. Morse, R. B. Makidon, L. E. Bergeron, S. Casertano, D. F. Figer, D. S. Acton, P. D. Atcheson, and M. J. Rieke, "Limits on routine wavefront sensing with NIRCam on JWST," Optical, Infrared, and Millimeter Space Telescopes 5487(1), pp. 909-917, SPIE, 2004.

3. F. Shi, D. C. Redding, J. J. Green, and C. M. Ohara, "Performance of segmented mirror coarse phasing with a dispersed fringe sensor: modeling and simulations," Optical, Infrared, and Millimeter Space Telescopes 5487(1), pp. 897-908, SPIE, 2004. 
4. M. J. Li, N. Acuna, E. Amatucci, M. Beamesderfer, R. A. Boucarut, S. Babu, S. Bajikar, A. J. Ewin, R. Fettig, D. E. Franz, L. Hess, R. Hu, M. D. Jhabvala, D. Kelly, T. T. King, G. Kletetschka, C. A. Kotechi, A. Kutyrev, J. P. Loughlin, B. A. Lynch, H. Moseley, B. Mott, B. Newell, L. Oh, D. A. Rapchun, C. Ray, C. Sappington, E. Schulte, S. Schwinger, W. Smith, S. Snodgrass, L. M. Sparr, R. Steptoe-Jackson, L. L. Wang, Y. Zheng, and C. A. Zincke, "Microshutter array development for the James Webb Space Telescope, publisher = SPIE," Micro- and Nanotechnology: Materials, Processes, Packaging, and Systems II 5650(1), pp. 9-16, 2005.

5. L. W. Huff, "NIRCam instrument optics," Cryogenic Optical Systems and Instruments XI 5904(1), p. 590404, SPIE, 2005.

6. J. T. Rayner, "Evaluation of a solid KRS-5 grism for infrared astronomy," Infrared Astronomical Instrumentation 3354(1), pp. 289-294, SPIE, 1998.

7. D. Charbonneau and D. Deming, "The Dynamics-Based Approach to Studying Terrestrial Exoplanets," ArXiv e-prints 706, June 2007.

8. J. J. Fortney, D. Saumon, M. S. Marley, K. Lodders, and R. S. Freedman, "Atmosphere, Interior, and Evolution of the Metal-rich Transiting Planet HD 149026b," The Astrophysical Journal 642, pp. 495-504, May 2006.

9. M. R. Swain, J. Bouwman, R. Akeson, S. Lawler, and C. Beichman, "The mid-infrared spectrum of the transiting exoplanet HD 209458b," ArXiv Astrophysics e-prints , Feb. 2007.

10. H. A. Knutson, D. Charbonneau, L. E. Allen, J. J. Fortney, E. Agol, N. B. Cowan, A. P. Showman, C. S. Cooper, and S. T. Megeath, "A map of the day-night contrast of the extrasolar planet HD 189733b," Nature 447, pp. 183-186, May 2007.

11. J. J. Fortney, C. S. Cooper, A. P. Showman, M. S. Marley, and R. S. Freedman, "The Influence of Atmospheric Dynamics on the Infrared Spectra and Light Curves of Hot Jupiters," The Astrophysical Journal 652 , pp. 746-757, Nov. 2006.

12. E. Rauscher, K. Menou, J. Y.-K. Cho, S. Seager, and B. M. S. Hansen, "Hot Jupiter Variability in Eclipse Depth," Astrophysical Journal Letters 662, pp. L115-L118, June 2007.

13. G. Chauvin, A.-M. Lagrange, C. Dumas, B. Zuckerman, D. Mouillet, I. Song, J.-L. Beuzit, and P. Lowrance, "A giant planet candidate near a young brown dwarf. Direct VLT/NACO observations using IR wavefront sensing," Astronomy and Astrophysics 425, pp. L29-L32, Oct. 2004.

14. P. Hartigan, S. Edwards, and R. Pierson, "Going Slitless: Images of Forbidden-Line Emission Regions of Classical T Tauri Stars Observed with the Hubble Space Telescope," The Astrophysical Journal 609, pp. 261-276, July 2004. 